REVIEW ARTICLE

\author{
Z. Rumboldt \\ W. Huda \\ J.W. All
}

\section{Review of Portable CT with Assessment of a Dedicated Head CT Scanner}

\begin{abstract}
SUMMARY: This article reviews a number of portable CT scanners for clinical imaging. These include the CereTom, Tomoscan, xCAT ENT, and OTOscan. The Tomoscan scanner consists of a gantry with multisection detectors and a detachable table. It can perform a full-body scanning, or the gantry can be used without the table to scan the head. The xCAT ENT is a conebeam CT scanner that is intended for intraoperative scanning of cranial bones and sinuses. The OTOscan is a multisection CT scanner intended for imaging in ear, nose, and throat settings and can be used to assess bone and soft tissue of the head. We also specifically evaluated the technical and clinical performance of the CereTom, a scanner designed specifically for neuroradiologic head imaging. The contrast performance of this scanner permitted the detection of 4-mm low-contrast lesions, and the limiting spatial resolution was 7 line pairs per centimeter. The measured volume of the CT dose index (CTDI $\left.{ }_{\text {vol }}\right)$ for a standard head CT scan was $41 \mathrm{mGy}(120 \mathrm{kV} / 14 \mathrm{mAs})$. All clinical images were of diagnostic quality, and the average patient effective dose was $1.7 \mathrm{mSv}$. We conclude that the CereTom portable CT scanner generates satisfactory clinical images at acceptable patient doses.
\end{abstract}

$\mathbf{P}$ ortable CT scanning can be both economically and clinically beneficial. ${ }^{1}$ Considering the clinical benefit, physicians can obviate the risks associated with intrahospital transport in situations in which portable CT is available for a patient in critical care. ${ }^{1-6}$ These risks are numerous and include the compromise of monitoring equipment, intravenous lines, and intubation tubes. ${ }^{5}$ In patients being transported for the specific purpose of a head CT, potential adverse events include hypotension, hypoxia, and increased intracranial pressure. ${ }^{5}$ The risk inherent in the transport of this patient population can reach $70 \%$ during transport to the CT suite of some hospitals. ${ }^{5}$ Waydhas reported that even in the setting of a well-trained transport team, adverse events still occurred $15 \%$ of the time. ${ }^{5}$ Moreover, despite these risks, the transport of a patient was still deemed clinically necessary, with a change of therapeutic management in $25 \%$ of imaged patients. Portable CT technology could also prove useful in the emergency department for evaluating patients suspected of stroke or head trauma, because decreasing the time it takes to obtain imaging improves the prognosis in these patients. ${ }^{7}$

Avoiding the transportation of patients in the intensive care unit (ICU) for the purpose of imaging has added benefits. Portable CT scanning with a machine dedicated to head imaging reduces the amount of time it takes before imaging results are available by eliminating transport time for a patient, which may be approximated at 50 minutes. ${ }^{1}$ In contrast, a portable CT scanning is completed in approximately $20 \mathrm{~min}$ utes from the time it was ordered. ${ }^{1}$

Introducing portable CT technology also serves to improve the utility of the stationary CT equipment of a hospital. ${ }^{1}$ This confers 2 particular advantages: First, an improvement in

From the Department of Radiology and Radiological Sciences (Z.R., W.H.) and College of Medicine (J.W.A.), Medical University of South Carolina, Charleston, SC.

Paper previously presented as a poster at: Annual Meeting of the American Society of Neuroradiology, May 31-June 5, 2008; New Orleans, La.

Please address correspondence to Zoran Rumboldt, MD, Department of Radiology and Radiological Science, Medical University of South Carolina, 96 Jonathan Lucas St, Charleston, SC 29425; e-mail: rumbolz@musc.edu

Indicates open access to non-subscribers at www.ajnr.org

DOI 10.3174/ajnr.A1603 work flow of standard scanners translates into faster imaging for non-ICU patients in a hospital, thereby improving their quality of care. Second, improving the use of other equipment contributes to the economic benefits gained with a portable CT machine. ${ }^{1}$

The economic advantages of this technology make it a viable option for improving patient care. A cost analysis done on the use of the CereTom (NeuroLogica, Danvers, Mass) at a level I trauma center calculated a return on investment of $169 \% .^{1}$ These calculations took into consideration the cost of the machine, $\$ 359,000$, and the single technician needed to operate it. The costs were weighed against those of personnel and equipment needed for the transport of a patient to and from the conventional scanner.

Several portable CT scanners are available for clinical imaging. These include the CereTom, the Tomoscan (Philips Medical Systems, Best, the Netherlands), the xCAT ENT (Xoran Technologies, Ann Abor, Mich), and the OTOscan (NeuroLogica). The Tomoscan scanner consists of a gantry with multisection detectors and a detachable table. It can perform a full-body scanning, or the gantry can be used without the table to scan the head. The xCAT ENT is a conebeam CT (CBCT) scanner that is intended for intraoperative scanning of cranial bones and sinuses. The OTOscan is a multisection CT (MSCT) scanner intended for imaging in ear, nose, and throat (ENT) settings and can be used to assess bone and soft tissue of the head.

In this review of portable CT scanners, we also specifically evaluated the technical and clinical performance of the CereTom in a head-to-head comparison with conventional CT scanners.

\section{Portable CT Scanners}

In discussing portable CT equipment, we will make a distinction between the MSCT and CBCT machines. In place of the multiple single-dimension detectors of a traditional MSCT scanner, a CBCT machine has a single volumetric flat panel detector used to acquire images in a single rotation of the gantry. ${ }^{8} \mathrm{CBCT}$ scanners produce a cone-shaped beam of radiation that is perceived by this single $2 \mathrm{D}$ flat panel. This creates a volume of data from which images are derived. ${ }^{9}$ In contrast, 


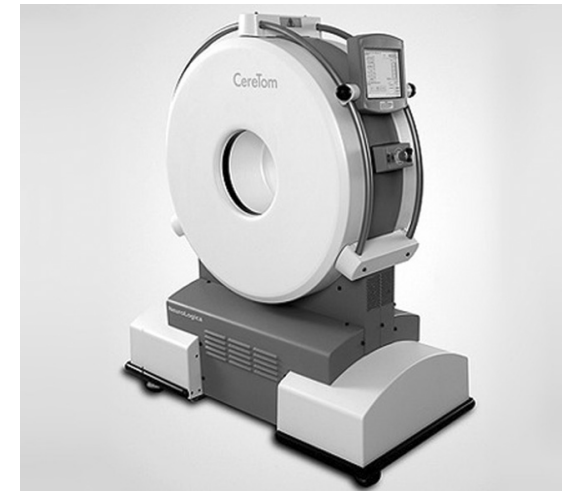

Fig 1. CereTom portable CT scanner

conventional MSCT scanners use a fan-shaped x-ray source that is registered in sequential sections, which are then stacked together to render the final sets of images. This difference has several implications.

The advantages of CBCT include a smaller scanner size, lower cost, decreased total time required for imaging, and decreased radiation. ${ }^{9}$ A CBCT scanner is typically about one fourth the price of a conventional scanner, though significant maintenance may undermine some of the financial advantages. ${ }^{9}$ CBCT does have a better high-contrast resolution than MSCT, yielding submillimeter details of bony structures. ${ }^{9,10}$ This, along with decreased metal artifacts, make CBCT machines ideal for use in dental and ENT settings where the visualization of small bones is of paramount importance. Portable CBCT is also being evaluated for several intraoperative and interventional applications and as a potential tool in airway analysis. ${ }^{9,11}$

The disadvantages of CBCT include limited options for setting kilovolt $(\mathrm{kV})$ and milliampere second (mAs), and the FOV is limited to either a spheric or cylindric shape. ${ }^{10}$ The size of the FOV for most machines is small, though some, such as the i-CAT (Imaging Sciences International, Hatfield, $\mathrm{Pa}$ ), claim that an FOV of $20 \times 25 \mathrm{~cm}$ may be obtained. ${ }^{9}$ However, an increase in the FOV with a CBCT scanner will result in increased noise, thereby degrading image quality. ${ }^{11}$ Compared with MSCT, CBCT has increased scatter radiation, leading to a reduced contrast-to-noise ratio. ${ }^{11} \mathrm{CBCT}$ produces a lower quality imaging of soft tissues and has difficulty with air-soft tissue interfaces, ${ }^{10}$ and, at the present time, has no intracranial application.

Several small CBCT scanners are available but, unlike the $\mathrm{xCAT}$, are not portable. They were created primarily for dental and orthodontic imaging, and if portability was advantageous, size would not preclude such a feature in future generations of CBCT equipment. ${ }^{9}$ Current smaller sized nonportable CBCT scanners include the following: the NewTom 3G (QR s.r.l., Verona, Italy), the i-CAT, the CB MercuRay (Hitachi Medical Systems, Twinsburg, Ohio), the 3D Accuitomo (J. Morita Mfg., Osaka, Japan), the Iluma (GE Healthcare, Waukesha, Wis), the Galileos (Sirona Dental Systems, Long Island City, NY), and the ProMax 3D (Planmeca, Helsinki, Finland).

\section{CereTom}

The CereTom (Fig 1), produced by NeuroLogica, attaches directly to a patient's bed and is designed for portable multisec- tion scanning of the head and neck. Although the company also suggests that the machine could be used for examination of the extremities, to our knowledge, there has been no investigation of this possible application. The CereTom has been deployed outside the hospital setting, with NeuroLogica providing a machine at National Football League games, the Indy 500 , and a professional boxing match, where its use helped to diagnose a postfight subdural hematoma. Less relevant product placement has afforded the scanner several cameos on medical television shows such as ER, Grey's Anatomy, and Diagnosis $X$.

The CereTom is significantly smaller than a traditional scanner, with a height of $153 \mathrm{~cm}$, a length of $134 \mathrm{~cm}$, and a width of $73 \mathrm{~cm}$. This machine weighs $<800 \mathrm{lb}(362.87 \mathrm{~kg})$ and requires a single technician for its transport. Eight $1.25-\mathrm{mm}$ wide detectors provide a $10-\mathrm{mm}$ collimated beam. ${ }^{12}$ Typical scanning parameters are $120 \mathrm{kV}, 7 \mathrm{~mA}$, and a scanning time of 2 seconds. Reconstructed section thicknesses are $1.25 \mathrm{~mm}$ in CT angiography, $5 \mathrm{~mm}$ for head scans, and $10 \mathrm{~mm}$ for perfusion imaging. ${ }^{12}$ Functionally, this machine can be used to perform CT scanning with and without contrast, CT angiography, CT perfusion, and CT fluoroscopy. It has potential application in several small-room environments, including clinics, critical care departments, departments of surgery, and emergency departments.

A cost analysis of portable CT scanning with the CereTom did show an economic benefit. ${ }^{1}$ Although prior portable CTs had been more expensive to implement, the size of the CereTom means fewer personnel are required to transport it. This, along with a comparatively lower cost (approximately $\$ 350,000)$, translates into a favorable return on investment.

\section{Tomoscan M}

The Tomoscan M, also named the Anatom 2000, is a CT unit produced by Philips, consisting of a table $(600 \mathrm{lb}, 272.16 \mathrm{~kg})$, a detachable gantry ( $1000 \mathrm{lb}, 453.59 \mathrm{~kg}$ ), an operator's console, and an optional power CT injector unit. It is capable of fullbody scanning and has a cost comparable with that of a conventional CT unit. ${ }^{13}$ The weight of the gantry requires 2 persons to transport it. The scanner is powered by rechargeable batteries that can be charged with a standard AC power supply. Section times can be set to 2, 4, or 6 seconds, with 2 and 4 seconds used most often. ${ }^{7}$ The Tomoscan $\mathrm{M}$ tube voltage can be set to 120 or $130 \mathrm{kV}$, and it has a maximum tube current of $50 \mathrm{~mA} .^{6}$ The scanner has 384 solid-state detectors. ${ }^{6}$ With scanning parameters of $120 \mathrm{kV}, 80 \mathrm{mAs}$, and a section thickness of $5 \mathrm{~mm}$, the CT dose index was calculated at $47.2 \mathrm{mGy}^{6}$

The Tomoscan has been evaluated for its potential applications in emergency departments, departments of surgery, and ICU settings. ${ }^{14-18}$ For cranial imaging, a clinical evaluation of this machine in comparison with conventional scanners demonstrated increased noise and artifacts, but the diagnostic value was no different from that of the comparison unit. ${ }^{7}$ When scanning patients in locations in which space is limited, gantry motion allows $356 \mathrm{~mm}$ of scanning length, so a head scan can be obtained without removing the patient from his or her bed. ${ }^{7,19}$ This is an important advantage for both patients in the ICU and intraoperative procedures. ${ }^{15,17}$ In facilities where this machine is available, cranial CT is typically the most commonly ordered study. ${ }^{18}$ This scanner has also been studied as a 


\begin{tabular}{|c|c|c|c|c|}
\hline & $\begin{array}{l}\text { Head without } \\
\text { Contrast }\end{array}$ & $\begin{array}{l}\text { Head with } \\
\text { Contrast }\end{array}$ & $\begin{array}{c}\text { CT } \\
\text { Angiography }\end{array}$ & $\begin{array}{c}\text { CT } \\
\text { Perfusion }\end{array}$ \\
\hline Kilovolt & 140 & 120 & 120 & 100 \\
\hline Milliampere & 7 & 7 & 7 & 6 \\
\hline Time/rotation (sec) & 2 & 2 & 1 & 1 \\
\hline Milliampere second & 14 & 14 & 7 & 6 \\
\hline Reconstruction algorithm & Smooth & Smooth & Standard & Standard \\
\hline Axial/helical & Axial & Axial & Helical & Axial \\
\hline Z-axis collimation & 1.25 & 1.25 & 1.25 & 1.25 \\
\hline No. of channels & 8 & 8 & 8 & 8 \\
\hline Table increment or table speed & 10 & 10 & 10 & N/A \\
\hline Effective pitch & 1 & 1 & 1 & $\mathrm{~N} / \mathrm{A}$ \\
\hline Reconstructed scan width & 5 & 5 & 1.25 & 10 \\
\hline
\end{tabular}

Note:-N/A indicates not applicable; ACR, American College of Radiology.

tool for bedside monitoring of cerebral blood flow with xenon-CT. ${ }^{16}$ Use of a portable CT scanner for this purpose has not been extensively evaluated, but it is likely that the diagnostic value is preserved. ${ }^{7}$ The Tomoscan has proved beneficial in intraoperative applications ranging from pediatric neurosurgery to surgery of the neck, sinuses, and temporal bones. ${ }^{14,17}$

The Tomoscan is less valuable for abdominal imaging, with significant limitations when compared with a stationary machine. ${ }^{19}$ The difference in image quality is clinically significant and is particularly detrimental to the negative predictive value of a study.

Use of the Tomoscan for thoracic imaging achieved adequate image quality. ${ }^{6}$ Soft-tissue windows were roughly comparable in quality with those in conventional scanners, whereas the quality of lung windows was decreased, due to the slower acquisition time of the portable scanner. Despite this, the scanner still produced viable thoracic imaging in the ICU setting.

A cost analysis of this machine concluded that its implementation would be more expensive than continuing to scan ICU patients with traditional scanners. ${ }^{13}$

\section{xCAT ENT}

The XCAT ENT scanner (Xoran Technologies) is designed for intraoperative scanning of the sinuses, skull base, and temporal bones. ${ }^{20}$ It is a portable scanner with a single flat panel detector that generates images with a single rotation of the gantry. Scanning is performed with the patient positioned supine. The machine weighs $520 \mathrm{lb}(235.87 \mathrm{~kg})$, and measures $32 \times 47 \times 60$ inches $(81.28 \times 119.38 \times 152.4 \mathrm{~cm})$. The xCAT uses a tube voltage of $120 \mathrm{kV}$ and a current of $6 \mathrm{~mA}$ and requires a scanning time of 20 seconds. ${ }^{20}$ Reconstructed views and $3 \mathrm{D}$ imaging are possible. The minimum section thickness is $0.4 \mathrm{~mm}$, and the FOV may be as large as $14 \times 24 \mathrm{~cm}^{20}$ Including the time required for image processing, a full scanning of the paranasal sinuses can be acquired in under 3 minutes. ${ }^{21}$ The XCAT is designed for intraoperative use, but similar portable $\mathrm{CBCT}$ machines could take on different roles in the near future. ${ }^{21}$

\section{OTOscan}

The OTOscan, also produced by NeuroLogica, is designed for ENT imaging. Unlike the CBCT machines used to scan bone and soft tissue of the head, this machine has multisection detectors rather than a single flat panel detector. The OTOscan is

$\begin{aligned} & \text { Table 2: CT number (Hounsfield unit) accuracy for the CereTom and } \\
& \text { the specific requirements in the ACR CT accreditation program }\end{aligned}$
\begin{tabular}{lcl} 
Measured \\
Material & (HU) & ACR CT Accreditation Requirements \\
\hline Air & -990 & Between -1005 and $-970 \mathrm{HU}$ \\
Polyethylene & -106 & Between -107 and $-87 \mathrm{HU}$ \\
Water & -1.7 & Between -7 and $+7 \mathrm{HU} \mathrm{( \pm 5} \mathrm{HU}$ preferred) \\
Acrylic & 119 & Between 110 and $130 \mathrm{HU}$ \\
Bone & 1060 & Between 850 and $970 \mathrm{HU}$ \\
\hline
\end{tabular}

Note:- HU indicates Hounsfield units.

not useful for intracranial imaging. With a patient in the seated position, it allows direct imaging in both coronal and axial planes, instead of generating coronal images from axial reconstructions. ${ }^{22}$ Viewing is possible in $2 \mathrm{D}, 3 \mathrm{D}$, and multiplanar reformations, and both the FOV and the scanning distance are adjustable. ${ }^{20}$ The scanner weighs just under $362 \mathrm{~kg}$, has a height of 60.3 inches $(153.62 \mathrm{~cm})$, a length of 52.7 inches $(133.85 \mathrm{~cm})$, and a width of 28.7 inches $(72.89 \mathrm{~cm}){ }^{22}$ The chair and workstation are separate from the gantry.

\section{CereTom Image Quality and Radiation Dose}

\section{Protocols}

Table 1 summarizes the protocols used for performing the American College of Radiology (ACR) CT accreditation tests for dose and image quality (ie, default parameters as supplied by the manufacturer).

\section{Image Quality}

To assess image quality, we used the ACR CT accreditation phantom to measure 5 key image-quality parameters: 1 ) CT number accuracy, 2) section thickness, 3) low-contrast performance, 4) uniformity, and 5) high-contrast resolution.

CT Number Accuracy. A CT number, also known as a Hounsfield unit, is directly related to the $\mathrm{x}$-ray attenuation of the material within each image pixel. CT numbers quantify the attenuation of any material relative to that of water, where the attenuation is taken to be equal to zero. Accordingly, positive Hounsfield unit values attenuate more than water, and negative values, less than water.

Table 2 shows the measured values for the CereTom and the specific requirements of CT number accuracy in the ACR CT accreditation program. ${ }^{23}$ All the measured values were within the expected range, except for bone. The bone 
Hounsfield unit value was slightly higher (10\%) than the upper limit specified in the ACR CT accreditation program. This minor discrepancy is of little clinical significance in neuroradiologic imaging.

Section Thickness. The ACR CT accreditation program requires the measurement of axial section widths, and those widths associated with helical imaging are generally not measured. Section-width measurements are obtained from the apparent size in images of discrete wires positioned on a ramp.

Image data were acquired with reconstructed section thicknesses of 3, 5, and $7 \mathrm{~mm}$. All measured section-thickness values were within $0.25 \mathrm{~mm}$ of the nominal section thickness value, which is well within the requirement of the ACR CT accreditation program, (ie, $<1.5 \mathrm{~mm}$ of the prescribed width).

Low-Contrast Detection Performance. Detection of lowcontrast lesions is a clinically relevant task that is primarily affected by the amount of noise in any CT image. CT is generally a quantum noise-limited imaging system, so the amount of noise is directly related to the total number of $\mathrm{x}$-ray photons used to create an image. The most important parameters that affect the number of $\mathrm{x}$-ray photons in CT are the $\mathrm{x}$-ray tube voltage (kilovolt), x-ray tube intensity (milliampere second), and the section thickness ( $\mathrm{T}$ ). Increasing kilovolt, milliampere second, and $\mathrm{T}$ will generally increase the number of photons and will, therefore, reduce the corresponding amount of noise in any reconstructed CT image. Because noise is directly related to the number of photons used to generate an image, it is also directly related to patient dose. In other words, more photons generally mean a lower level of noise, improved low-contrast detection performance, and higher patient doses.

For this test, we used the adult head protocol without contrast (Table 1). The low-contrast phantom has 5 groups of 4 cylinders with varying diameters. Each cylinder has a CT number that differs by $6 \mathrm{HU}$ from the surrounding background. The diameters of the 5 groups of cylinders are $6,5,4,3$, and 2 mm.

Three sets (ie, 4 cylinders in each set) of low-contrast lesions with sizes of 6,5 , and $4 \mathrm{~mm}$ were visible in the reconstructed image. This level of performance surpasses the minimum performance required by the ACR CT accreditation program, which requires that all four $6-\mathrm{mm}$ rods be clearly visible.

Uniformity. Proper calibration of a CT system should result in an image of a phantom that will appear uniform and without any visible artifacts. Image uniformity is obtained by comparing the mean CT number at the phantom center with the mean values at the 4 edge positions. Mathematically, image uniformity is defined as (Average Hounsfield Unit at Center) - (Average Hounsfield Unit at Edge).

We used the head CT protocol, rather than the abdominal protocol normally required in the accreditation program (because CereTom is a head-only CT scanner). The maximum nonuniformity was $2.8 \mathrm{HU}$, and no image artifacts were visible on the reconstructed images. This level of performance is much better than that required by the ACR CT accreditation program, in which the difference between the edge and center mean Hounsfield unit values should be $<5 \mathrm{HU}$ at all 4 edge positions.

High-Contrast Resolution. A high-contrast-resolution image is generated from a line-pair phantom. The phantom contains 8 sets of inserts that correspond to spatial frequencies of $4,5,6,7,8,9,10$, and 12 line pairs per centimeter $(\mathrm{lp} / \mathrm{cm})$.

We used the adult head (without contrast) as listed in Table 1 , which uses the smooth image reconstruction filter. The reconstructed CT images could depict at least $7 \mathrm{lp} / \mathrm{cm}$. The ACR $\mathrm{CT}$ accreditation program requires that the $5-\mathrm{lp} / \mathrm{cm}$ bar pattern be resolved for the adult abdomen protocol and that the $6-\mathrm{lp} / \mathrm{cm}$ bar pattern be resolved for a high-resolution chest protocol. The resolution performance of the CereTom is satisfactory.

\section{Radiation Dose}

CT dose index values are obtained by using an axial (not helical) scan, and the technique factors for a routine head CT scan (ie, kilovolt, milliampere, exposure time, beam width, etc). A 16-cm-diameter acrylic phantom was used to measure the CTDI with a 100 -mm-long pencil ionization chamber. Technique factors used to perform routine head CT scans (120 $\mathrm{kV} / 14 \mathrm{mAs}$ ) with an effective pitch of 1 (Table 1 ) resulted in a volume CTDI of $41 \mathrm{mGy}$.

The ACR CT accreditation program initially required the value of CTDI ${ }_{\mathrm{vol}}$ to be $<60 \mathrm{mGy}$. If the value of CTDI $\mathrm{vol}_{\mathrm{vo}}$ for a facility exceeded $60 \mathrm{mGy}$, known as the "reference dose," the facility was notified of this fact with no further action being taken. The ACR CT accreditation dose requirements have recently been changed, and the reference dose value has been increased to $75 \mathrm{mGy}$ for head CT scans. In addition, facilities that exceed 80 mGy for the CTDI ${ }_{\mathrm{vol}}$ in adult head examinations "fail" the accreditation program and must reduce their doses if they wish to attain the accredited status.

Patient effective doses were estimated by computing the dose-length product (DLP) (ie, CTDI $\times$ scanning length), which was converted to an effective (E) dose by using an $\mathrm{E} / \mathrm{DLP}$ conversion factor of $2.2 \mu \mathrm{Sv} / \mathrm{mGy} \mathrm{cm}$. A typical scanning length is $\sim 19 \mathrm{~cm}$, corresponding to an average DLP of $770 \mathrm{mGy} \mathrm{cm}$. The resultant average patient effective dose for a routine head CT examination on the CereTom is thus $\sim 1.7$ $\mathrm{mSv}$. This radiation exposure is typical for head examinations performed on any CT scanner where adults receive between 1 and $2 \mathrm{mSv}^{24}$

\section{Clinical Performance}

The mobile CT scanner was commissioned in November 2007 , and during the first year, $>200$ routine adult head CT examinations were performed without the administration of iodinated contrast.

Two observers in consensus evaluated the head CT images from 60 consecutive patients in whom comparison studies had been acquired on standard clinical scanners (16- or 64-section MSCT) within 48 hours before or after imaging with the portable scanner. Scans were excluded if prominent artifacts from external devices prevented a clear assessment of standard CT images, portable CT images, or both. The images were ranked at 3 different levels (middle cerebellar peduncles, basal ganglia, and centrum semiovale) for streak artifacts, noise, gray/white matter differentiation, visualization of lesions, and overall image quality. A 3-point scale was used for evaluations: 1) poor, 2) acceptable, 3) good. Direct comparison between the 2 scans was also performed by selecting the study with better overall 

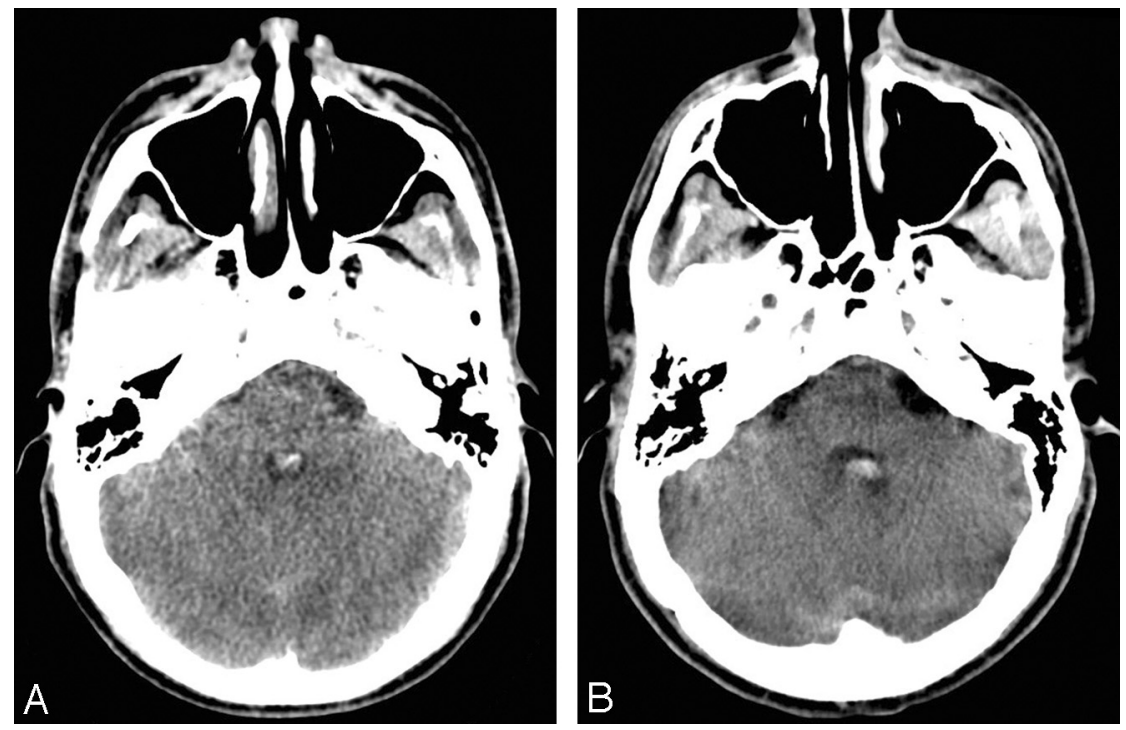

Fig 2. Axial CT images at the level of the middle cerebellar peduncles show blood clot within the fourth ventricle. $A$ Image from the portable CereTom scanner. $B$, Image at a level comparable with that in $A$ acquired on a standard clinical scanner 12 hours later. Note relatively increased noise, however, with clear visualization of the hyperattenuated lesion with the portable scanner.
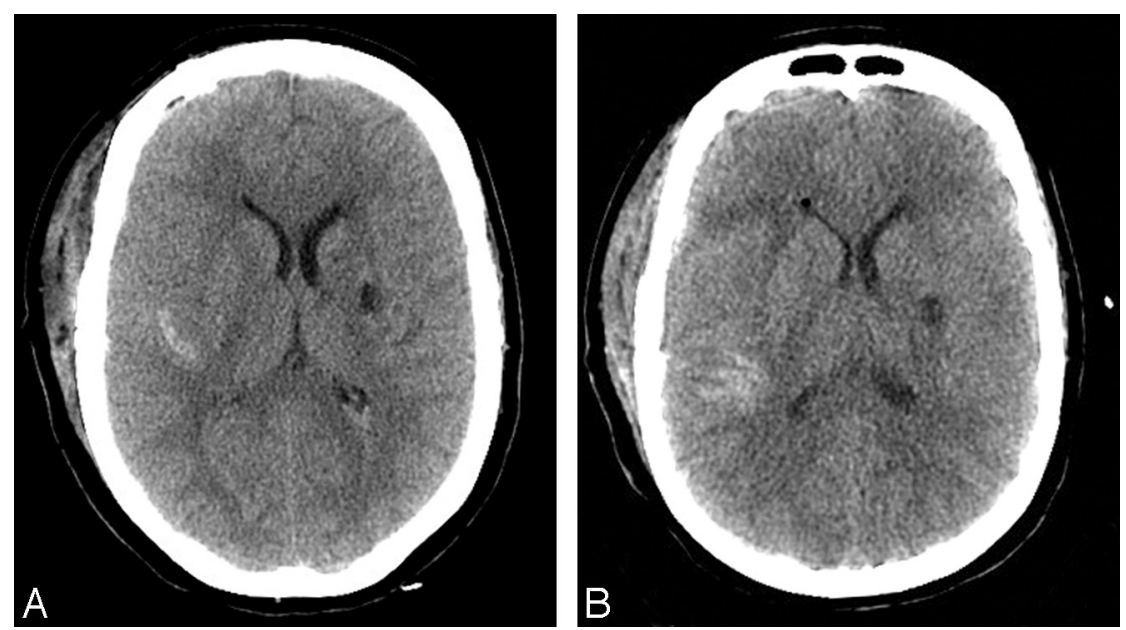

Fig 3. Axial CT images at the basal ganglia level show subarachnoid hemorrhage on the right and a chronic lacunar infarct on the left. $A$, Image from a standard CT scanner. $B$, Image at a level comparable with that in $A$ acquired on the portable scanner 16 hours later. There is clear visualization of both the hyperattenuated and hypoattenuated lesions with the portable scanner, despite an increased amount of noise and streak artifacts
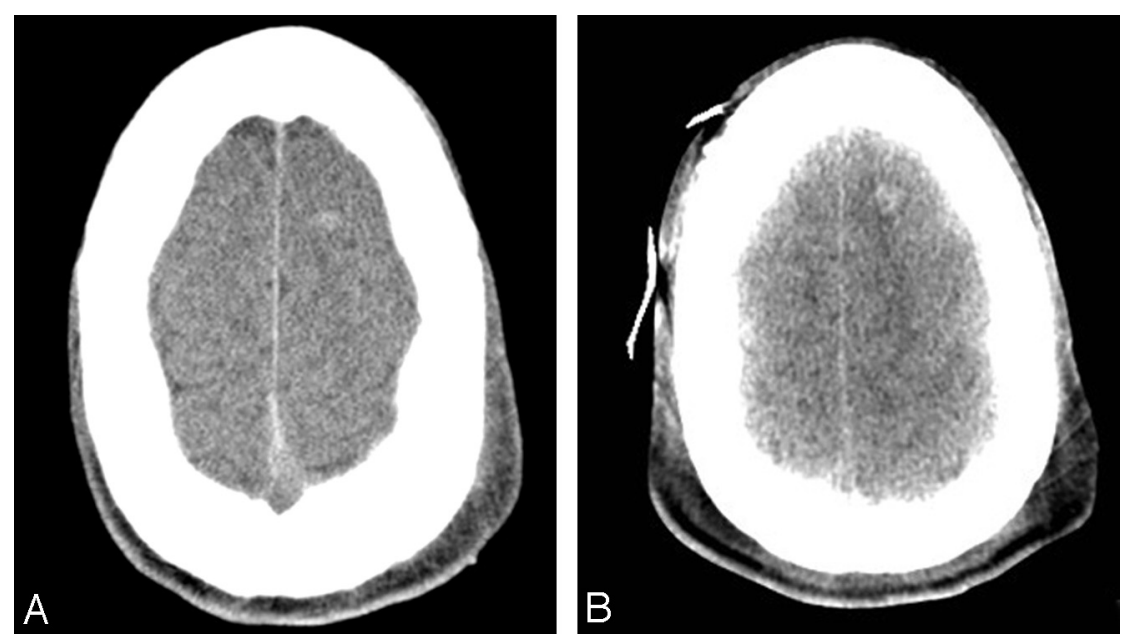

Fig 4. Axial CT images at the centrum semiovale level show a small left frontal hemorrhage corresponding to shear injury. $A$, Image from a standard scanner. $B$, Image at a level comparable with that in $A$ acquired on the portable scanner 8 hours later. Slightly better visualization of the hyperattenuated lesion with the portable scanner is likely due to interval clot retraction and development of perilesional edema.

image quality. Statistical analysis was performed by using the Wilcoxon test to derive a $\mathrm{z}$-score.

All the acquired studies were of diagnostic quality, and all the hyperattenuated and hypoattenuated lesions were clearly visualized in all patients (Fig 2). Of the 60 reviewed patients, 59 had positive findings, none of which were missed on the portable CT images. Mobile scanner images had, on average, rel- atively decreased contrast between gray and white matter and contained higher levels of noise and artifacts (Figs 2-4).

The distinction between the gray and white matter was characterized by an average score of 2.75 for the portable scanner versus 2.9 for the stationary scanner at the centrum semiovale $(z=4.02, P=.0276), 2.82$ versus 2.9 at the basal ganglia $(z=1.14, P=.128)$, and 2.68 versus 2.85 at the middle cere- 
bellar peduncles $(z=2.07, P=.019)$. Comparison of the image noise showed an average score of 2.38 for CereTom images versus 2.95 for images from standard CT scanners $(z=$ 5.02, $P<.0001)$ at the centrum semiovale, 2.35 versus 2.9 at the basal ganglia $(z=5.02, P<.0001)$, and 2.13 versus 2.87 at the middle cerebellar peduncles $(z=5.58, P<.0001)$. Evaluation of streak artifacts demonstrated an average score of 2.57 with the portable scanner versus 2.83 with the standard scanners $(z=2.61, P=.005)$ at the centrum semiovale, 2.21 versus 2.67 at the basal ganglia $(z=3.55, P=.0002)$, and 1.8 versus 2.53 at the middle cerebellar peduncles $(z=4.66, P<.0001)$. Of note, artifacts that appeared hyperattenuated on conventional CT were observed to be hypoattenuated on the corresponding portable CT images.

Direct comparison of CereTom images with those from a standard clinical CT scanner showed that the latter were preferred in $98 \%$ of the cases for imaging at the level of the centrum semiovale and the middle cerebellar peduncles. The standard CT scanner was preferred in $93 \%$ of cases for imaging at the level of the basal ganglia. Nonetheless, all of the portable scanner studies were considered to be adequate for diagnostic purposes. CT angiography and CT perfusion were not performed in a sufficient number of patients to allow a proper evaluation.

\section{Discussion}

Portable CT technology provides a better way to obtain diagnostic imaging in the critical care patient population. ${ }^{1-6}$ Imaging of these patients is considered valuable enough to warrant the increase in both cost and risk associated with their transport. ${ }^{5}$ Some facilities have installed a CT machine in a suite connected to the ICU, and though this solution does help to improve patient care for patients in the ICU, it is a costly solution that still requires a patient to be moved from his or her room, albeit to an area in much closer proximity. ${ }^{4}$ Portable CT equipment avoids the risk of transport and hence provides an economic benefit for providing this form of patient care. ${ }^{1}$ Although abdominal imaging on portable CT has shown significant diagnostic limitations, a CT head examination performed on transportable equipment has proved quite useful. ${ }^{1,7,19}$ Moreover, at a facility with a full-sized portable CT scanner, imaging of the head was the most commonly ordered examination. ${ }^{2}$ Reducing the patient volume scanned on the standard CT machines would benefit other patients in the form of improved availability of that equipment. Thus, a transportable device for dedicated head CT imaging provides a financially viable means to solve a commonly encountered problem. Further investigation is needed to determine an algorithm of how a facility should best coordinate the use of both its portable and stationary CT equipment.

Smaller CT equipment using CBCT is being used in dental, orthodontic, ENT, and intraoperative settings. ${ }^{9,10,17}$ CBCT imaging is also being obtained via incorporating a volumetric detector (PaxScan 4030CB; Varian Imaging Products, Palo Alto, Calif) onto a PowerMobil portable C-arm scanner (Siemens, Erlangen, Germany). ${ }^{25}$ This setup is currently being evaluated for use in a more extensive range of procedural applications, including a promising role in image-guided surgery of the head and neck. ${ }^{25}$ Other areas under investigation include image guidance of brachytherapy; external beam radia- tion therapy; and applications in spinal, orthopedic, thoracic, and abdominal surgeries. ${ }^{11}$ The use of this technology for interventional radiologic procedures, including neurointerventional ones, is also being assessed. ${ }^{11,25}$ It is unlikely, however, that this technology will be used for the same diagnostic imaging purposes as standard clinical MSCT scanners because it cannot produce the same image quality. Portable CBCT has much to offer in the way of interventional and interoperational environments, and its future success lies not in its ability to replace MSCT but rather to augment it.

With respect to the CereTom portable scanner that we evaluated, the purpose of our investigation was twofold: to use portable CT scanning without exposing a patient to an increase in radiation dose and to ensure that the choice of portable CT scanning does not compromise the diagnostic quality of the obtained images. A reduction in radiation dose decreases exposure not just to the patients but also to other people. This is important for enabling use of the machine in a wider range of inpatient environments. For example, in an ICU setting where patients are in close proximity, increasing the radiation dose might be prohibitive due to concerns about the radiation exposure of staff and other patients. We found that the quality of images acquired with a level of radiation comparable to that of standard scanners was reduced. However, although the overall image quality was decreased, the diagnostic accuracy and reliability was unchanged.

\section{Conclusions}

Portable CT scanning has a role in the management of patients in critical care and is a financially viable means for improving patient care. Volumetric conebeam technology is currently not feasible for brain imaging but continuing advances will likely see it used in a broader range of applications. Head CT images acquired with the CereTom portable scanner are satisfactory for clinical use at an effective dose of $1.7 \mathrm{mSv}$ per scan and were found to be diagnostically accurate in all cases.

\section{References}

1. Masaryk T, Kolonick R, Painter T, et al. The economic and clinical benefits of portable head/neck CT imaging in the intensive care unit. Radiol Manage 2008; 30:50-54

2. McCunn M, Mirvis S, Reynolds HN, et al. Physician utilization of a portable computed tomography scanner in the intensive care unit. Crit Care Med 2008;28:3808-13

3. Smith I, Fleming S, Cernaianu A. Mishaps during transport from the intensive care unit. Crit Care Med 1990;18:278-81

4. Teichgräber UK, Pinkernelle J, Jürgensen JS, et al. Portable computed tomography performed on the intensive care unit. Intensive Care Med 2003;29: 491-95

5. Waydhas C. Intrahospital transport of critically ill patients. Crit Care 1999; 3:R83-89

6. White CS, Meyer CA, Wu J, et al. Portable CT: assessing thoracic disease in the intensive care unit. AJR Am J Roentgenol 1999;173:1351-56

7. Matson MB, Jarosz JM, Gallacher D, et al. Evaluation of head examinations produced with a mobile CT unit. Br J Radiol 1999;72:631-36

8. Gupta R, Grasruck M, Suess C, et al. Ultra-high resolution flat-panel volume CT: fundamental principles, design architecture, and system characterization. Eur Radiol 2006;16:1191-205

9. Kau CH, Richmond S, Paloma JM, et al. Three-dimensional cone beam computerized tomography in orthodontics. J Orthod 2005;32:282-93

10. Loubele M, Maes F, Jacobs R, et al. Comparative study of image quality for MSCT and CBCT scanners for dentomaxillofacial radiology applications. $R a-$ diat Prot Dosimetry 2008;129:222-26

11. Orth RC, Wallace MJ, Kuo MD. C-arm cone-beam CT: general principles and technical considerations for use in interventional radiology. J Vasc Interv Radiol 2008;19:814-20

12. CereTom Portable CT Scanner. NeuroLogica Website. Available at: http://www. 
neurologica.com/images//ceretom\%20brochure\%202009.pdf. Accessed February 23, 2009

13. Mayo-Smith WW, Rhea JT, Smith WJ, et al. Transportable versus fixed platform CT scanners: comparison of costs. Radiology 2003;226:63-68

14. Butler WE, Piaggio CM, Constantinou C, et al. A mobile computed tomographic scanner with intraoperative and intensive care unit applications. Neurosurgery 1998;42:1304-10

15. Gwinn R, Cleary K, Medlock M. Use of a portable CT scanner during resection of subcortical supratentorial astrocytomas of childhood. Pediatr Neurosurg 2000;32:37-43

16. Hillman J, Sturnegk P, Yonas $\mathrm{H}$, et al. Bedside monitoring of CBF with xenon-CT and a mobile scanner: a novel method in neurointensive care. $\mathrm{Br} J$ Neurosurg 2005;19:395-401

17. Mattox DE, Mirvis SE. Intraoperative portable computed tomography scanning: an adjunct to sinus and skull base surgery. Otolaryngol Head Neck Surg 1999;1201:776-80

18. Mirvis SE. Use of portable CT in the R Adams Cowley Shock Trauma Center: experiences in the admitting area, ICU, and operating room. Surg Clin North Am 1999;79:1317-30

19. Maher MM, Hahn PF, Gervais DA, et al. Portable abdominal CT: analysis of quality and clinical impact in more than $\mathbf{1 0 0}$ consecutive cases. AJR Am J Roentgenol 2004;183:663-70

20. xCAT ENT Technical Specifications. Xoran Technologies Website. Available at: http://www.xorantech.com/contentHTML/xcatent_techspecs.php. Accessed February 23, 2009

21. Chennupati SK, Woodworth BA, Palmer JN, et al. Intraoperative IGS/CT updates for complex endoscopic frontal sinus surgery. ORL J Otorhinolaryngol Relat Spec 2008;70:268-70. Epub 2008 May 19

22. An Evolution in ENT In-Office Scanning: CereTom OTOscan. NeuroLogica Website. Available at: http://www.neurologica.com/images/literature/1nl3100-102\%20rev03.pdf. Accessed February 23, 2009

23. McCollough $\mathrm{CH}$, Bruesewitz MR, McNitt-Gray MF, et al, for the American College of Radiology. The phantom portion of the American College of Radiology (ACR) computed tomography (CT) accreditation program: practical tips, artifact examples, and pitfalls to avoid. Med Phys 2004;31:2423-42

24. Mettler FA Jr, Thomadsen BR, Bhargavan M, et al. Medical radiation exposure in the U.S. in 2006: preliminary results. Health Phys 2008;95:502-07

25. Daly MJ, Siewerdsen JH, Moseley DJ, et al. Intraoperative cone-beam CT for guidance of head and neck surgery: assessment of dose and image quality using a C-arm prototype. Med Phys 2006;33:3767-80 